\title{
The NEXT-1 (Next generation pErsonalized tX with mulTi-omics and preclinical model) trial: prospective molecular screening trial of metastatic solid cancer patients, a feasibility analysis
}

\author{
Seung Tae Kim ${ }^{1, *}$, Jeeyun Lee ${ }^{1, *}$, Mineui Hong ${ }^{2,3, *}$, Kyunghee Park ${ }^{4,5, *}$, Joon Oh Park ${ }^{1}$, \\ Tae Jin Ahn ${ }^{3,4}$, Se Hoon Park ${ }^{1}$, Young Suk Park ${ }^{1}$, Ho Yeong Lim ${ }^{1}$, Jong-Mu Sun ${ }^{1}$, \\ Jin Seok Ahn', Myung-Ju Ahn', Hee Cheol Kim ${ }^{6}$, Tae Sung Sohn', Dong Il Choi', \\ Jong Ho Cho ${ }^{8}$, Jin Seok Heo', Wooil Kwon', Sang Won Uhm ${ }^{6}$, Hyuk Lee ${ }^{10}$, \\ Byung-Hoon $\mathrm{Min}^{10}$, Sung No $\mathrm{Hong}^{10}$, Duk Hwan Kim ${ }^{5,11}$, Sin Ho Jung ${ }^{12}$, \\ Woongyang Park ${ }^{4,5}$, Kyoung-Mee Kim ${ }^{2,3}$, Won Ki Kang ${ }^{1}$, Keunchil Park ${ }^{1,2}$

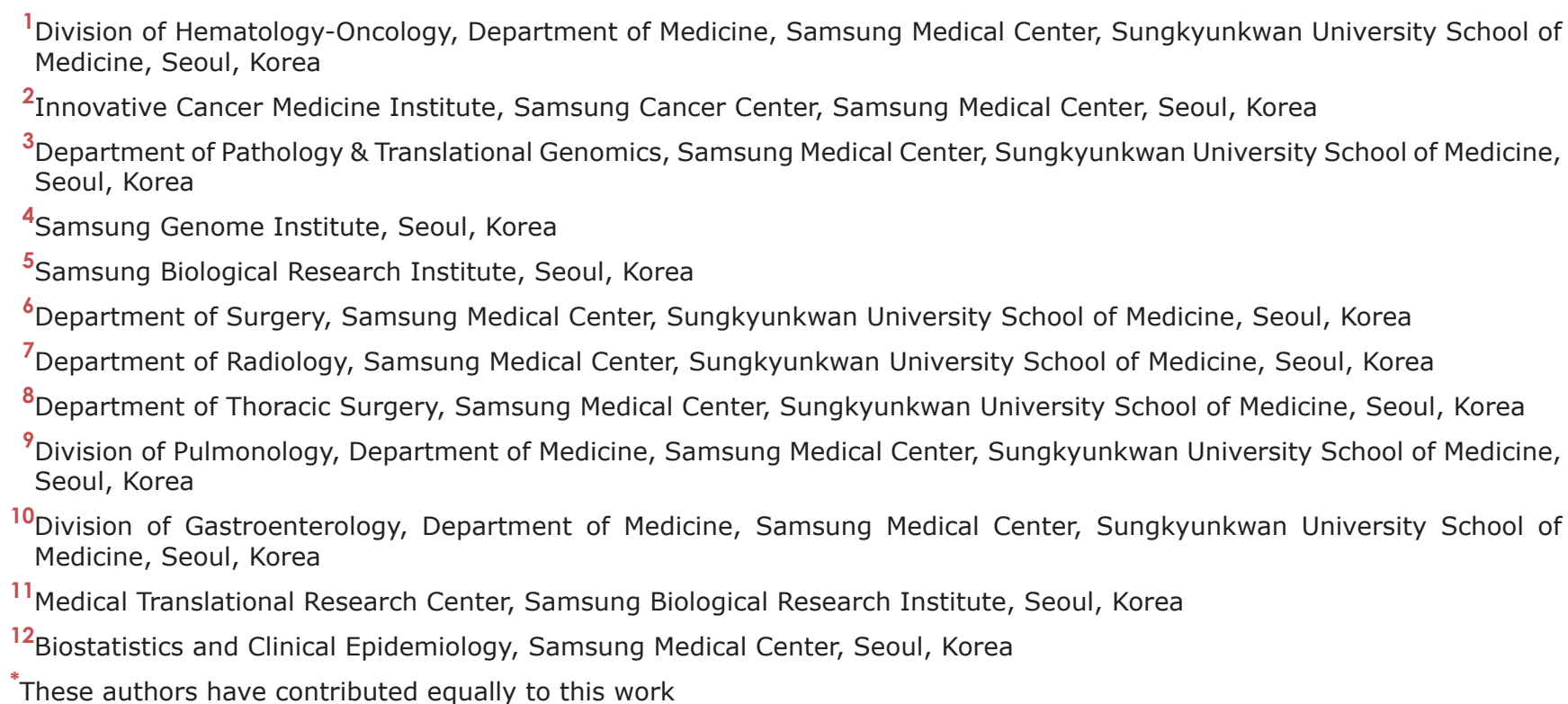

*These authors have contributed equally to this work \\ Correspondence to: \\ Won Ki Kang, e-mail: wkkang@skku.edu \\ Kyoung-Mee Kim, e-mail: kkmkys@skku.edu \\ Keunchil Park, e-mail: kpark@skku.edu \\ Keywords: molecular profiling, genome, ampliseq \\ Received: July 01, $2015 \quad$ Accepted: August 27, $2015 \quad$ Published: September 09, 2015
}

\section{ABSTRACT}

We conducted a prospective genomic screening trial with high throughput sequencing and copy number variation (CNV) assay, and immunohistochemistry array in metastatic solid cancer patients. We used Ion AmpliSeq Cancer Hotspot Panel v2 and nCounter Copy Number Variation Assay ( 21 genes) to identify molecular targets for potential matched therapy. Metastatic solid tumor patients were prospectively consented for molecular profiling tests. The primary outcome for this trial was the feasibility of molecular tests and response rate (matched vs non-matched treatment). Between November 2013 and August 2014, a total of 428 metastatic solid tumor patients were enrolled on to this study. The mutational profiles were obtained for $407(95.1 \%)$ patients. CNV 21-gene assays were successfully performed in $281(65.7 \%)$ of 428 patients. Of the 407 patients with molecular profiling results, 
$342(84.0 \%)$ patients had one or more aberrations detected. Of the 342 patients, 103 patients were matched to molecularly targeted agents in the context of clinical trials or clinical practice. The response rate was significantly higher in the genomematched treated group for gastrointestinal/hepatobiliary/rare tumors (matched vs non-matched treatment, $42.6 \%$ vs $24.3 \%, P=.009$ ) and lung cancer cohort (matched vs non-matched treatment, $61.2 \%$ vs $28.6 \%<P=.001$ ) when compared with the non-matched group. In this trial, we demonstrate that genome-matched treatment based on molecular profiling result in better treatment outcome in terms of response rate.

\section{INTRODUCTION}

With the advent of molecular sequencing era in medical oncology, upfront high-throughput genomic profiling of patient's tumor specimen is now becoming part of clinical trials or clinical research. Furthermore, systematic efforts to characterize the cancer genome constantly add genome alterations to the compilation of potentially actionable genomic alterations [1-7]. Currently, high-throughput genomic sequencing is not conducted routinely in the practice of medical oncology in most cancer centers. There may be several reasons for hindering from rapid application of clinical sequencing in oncology patients: 1) There is a discrepancy between genomic sequencing results and available matched drugs to the sequence in the clinic; 2) the clinical implication of genomic sequencing in each cancer type is not fully understood; 3) currently, unless a master protocol is present for "umbrella" genomic sequencing project which is simultaneously aligned with several matched drugs, the time from target identification through next-generation sequencing (NGS) and the actual application of the drug takes relatively long period of time.

Nevertheless, there have been previous attempts on molecular profiling of patients' tumors to find potential targets and provide matched trials to the patients. One of the early trials was a pilot study using molecular profiling of 86 patients' tumors of which 66 of them were provided of matched drugs [8]. One of the largest prospective trials was reported in 2012 which analyzed 1,144 patients with a pool of matched therapy available once genetic aberration is identified. Of 1,144 patients analyzed, $460(40.2 \%)$ had one or more aberration and 175 patients received matched therapy either in clinical trial or as clinical practice [9]. Based on their study, matched targeted therapy was associated with longer time-to-failure compared with their prior systemic therapy in patients with 1 mutation (5.2 vs. 3.1 months, respectively; $P<.0001$ ).

To address the ongoing challenges in actively incorporating genomics in the diagnosis and treatment of oncology patients, we conducted a prospective NEXT-1 trial with combinatorial approach encompassing somatic mutations, and a panel of CNVs. In this trial, we have utilized 1) the Ion Torrent AmpliSeq Cancer Panel (50 genes) to survey somatic mutations; 2) nanostring copy number variations (CNVs) to survey 21 actionable gene amplifications. The NGS platforms used in this study (AmpliSeq) relies on non-optical detection of hydrogen ions in a semiconductor device [10] and is able to detect 2,855 oncogenic mutations in 50 commonly mutated genes. We and others have shown that AmpliSeq is a highly feasible platform, particularly because it is associated with a low failure rate when using FFPE tissue specimens [11-14].

The primary objectives of this study were to survey the feasibility of genomic profiling in oncology patients and to compare response rate in matched treatment group versus non-matched conventional treatment group in the NEXT-1 trial. Herein, we report the feasibility of the genomic profiling for patients with metastatic cancer who were enrolled in the prospective master protocol study.

\section{RESULTS}

\section{Patient characteristics and molecular aberrations}

Between November 2013 and August 2014, 428 patients enrolled in this study (NEXT-1, $N=231$; LUNG PERSEQ, $N=98$ ). Table 1 provides baseline patient characteristics. All patients were Korean. The most frequent cancer types were gastric cancer (GC; $n=133,31.1 \%$ ), followed by non-small cell lung cancer (NSCLC; $n=99,23.1 \%$ ), colorectal cancer (CRC; $n=60$, $14 \%$ ), hepatocellular carcinoma (HCC; $n=21,6 \%$ ), sarcoma $(n=25,5.8 \%)$, gastroentro-pancreatic (GEP) neuroendocrine tumor $(n=15,3.5 \%)$, melanoma $(n=12$, $2.9 \%)$, cholangiocarcinoma $(n=14,3.3 \%)$, and pancreatic adenocarcinoma $(n=9,2.1 \%)$.

The study scheme is outlined in Fig 1 . Of 428 patients, 21 patients (all FFPE) did not enter the genomic analysis due to low tumor cellularity $(<10 \%)$ (Fig 1$)$. The mutational profiles were obtained in 407 (95.1\%) patients using Ampliseq. However, both Ampliseq and CNV 21-gene assays were successfully performed in $281(65.7 \%)$ of 428 patients. $237(58.2 \%)$ specimens were from FFPE tissues, and $170(41.8 \%)$ specimens were from fresh tumor tissues. Most fresh biopsies were obtained from endoscopic/colonoscopic/liver or bronchoscopic biopsies. The median time between biopsy and molecular results was 21 days (range, 17-28 days). Of the 407 patients with cancer panel data available, 
Table 1: Patient Characteristics

\begin{tabular}{|c|c|c|}
\hline Characteristics & No. of Patients $(N=428)$ & $\%$ \\
\hline \multicolumn{3}{|l|}{ Sex } \\
\hline Female & 175 & 40.8 \\
\hline Male & 253 & 59.2 \\
\hline \multicolumn{3}{|l|}{ Age. years } \\
\hline Median & 56 & \\
\hline Range & $18-82$ & \\
\hline \multicolumn{3}{|l|}{ ECOG PS } \\
\hline $0-1$ & 415 & 96.9 \\
\hline$\leq 2$ & 13 & 3.1 \\
\hline \multicolumn{3}{|l|}{ Tumor Types } \\
\hline Gastric adenocarcinoma & 133 & 31.1 \\
\hline Non-small cell lung cancer & 94 & 22.0 \\
\hline Colorectal adenocarcinoma & 60 & 14.0 \\
\hline Soft tissue sarcoma & 25 & 5.8 \\
\hline Hepatocellular carcinoma & 21 & 4.9 \\
\hline Gastroenteropancreatic neuroendocrine tumor & 15 & 3.5 \\
\hline Cholangiocarcinoma & 14 & 3.3 \\
\hline Melanoma & 12 & 2.8 \\
\hline Pancreatic adenocarcinoma & 9 & 2.1 \\
\hline Small cell lung cancer & 5 & 1.2 \\
\hline Gall bladder cancer & 5 & 1.2 \\
\hline Metastatic carcinoma of unknown origin & 4 & 0.9 \\
\hline GIST* & 4 & 0.9 \\
\hline Thymic carcinoma & 4 & 0.9 \\
\hline Ampulla of Vater cancer & 3 & 0.7 \\
\hline Esophageal squamous carcinoma & 3 & 0.7 \\
\hline Ovarian cancer & 3 & 0.7 \\
\hline Renal cell carcinoma & 3 & 0.7 \\
\hline Non-melanoma skin cancer & 3 & 0.7 \\
\hline Bladder cancer & 2 & 0.5 \\
\hline Uterine/cervix cancer & 2 & 0.5 \\
\hline Adrenocortical carcinoma & 1 & 0.2 \\
\hline Duodenal adenocarcinoma & 1 & 0.2 \\
\hline Glottic cancer & 1 & 0.2 \\
\hline Peritoneal mesothelioma & 1 & 0.2 \\
\hline Metastatic & 428 & 100.0 \\
\hline
\end{tabular}

*Gastrointestinal stromal tumors 


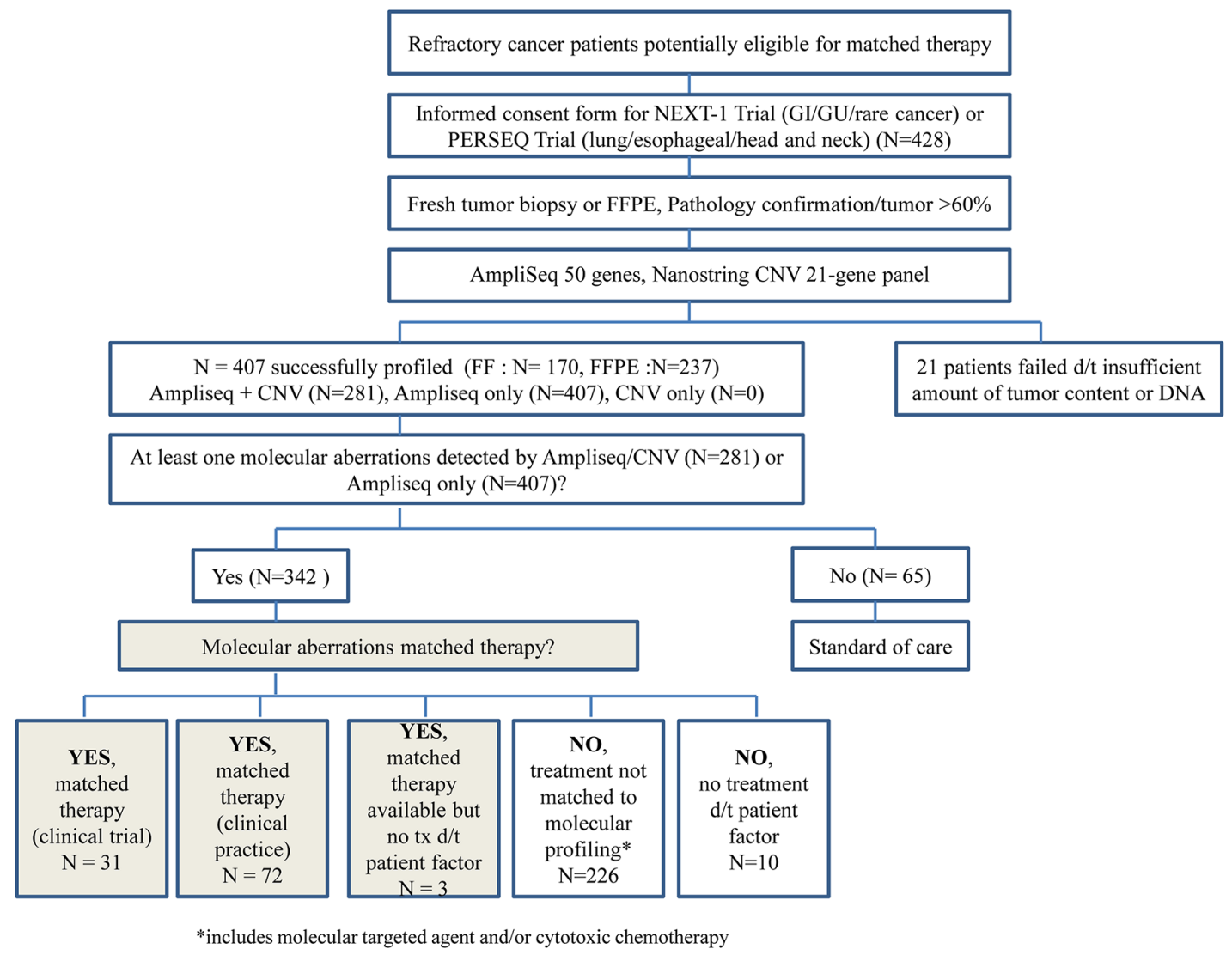

Figure 1: The Study Scheme.

$342(84.0 \%)$ patients had one or more aberrations detected (Table 2). Mutations were commonly detected in the following genes: TP53 (54.5\%), KRAS (14.5\%), EGFR (11.8\%), SMARCBI (10.3\%), PIK3CA (10.3\%), PTEN (6.4\%), and APC (5.8\%), as shown in Fig $2 \mathrm{~A}$ and $2 \mathrm{~B}$. Most of the genetic aberrations were somatic mutations identified from cancer panel and only 46 out of 281 cases $(16.4 \%)$ had one or more CNV detected through the CNV 21-gene assay (Table 2, Fig 2B). The most frequently detected amplifications were $M E T$ (2.1\%) and MDM2 (2.1\%), followed by EGFR (1.8\%), CDK4 (1.8\%), HER2 (1.8\%), KRAS (1.8\%), CCNE1 $(1.4 \%)$, and FGFR2 (1.4\%), as shown in Figure 2C.

In metastatic $\mathrm{GC}$ patients, mutations were frequently detected in TP53 (43.9\%), PIK3CA (12.1\%), SMARCBI (8.1\%), ALK (7.3\%), and PTEN (6.5\%), as shown in Supplementary Fig $1 \mathrm{~A}$ and in our previous study. ${ }^{11}$ For NSCLC, TP53 (90.4\%), EGFR (48.9\%), SMARCBI (8.5\%), PTEN (7.4\%), and KRAS (7.4\%) were frequently mutated (Supplementary Fig 1B). In metastatic CRC patients, the most frequently observed mutations were TP53 (62.1\%), KRAS (50.0\%), APC (31.0\%), PIK3CA (17.2\%), SMAD4 (13.8\%), SMARCB1 (12.1\%), BRAF (8.6\%), PTEN (5.1\%), MET (5.1\%), and FBXW7 (5.1\%) (Supplementary Fig 1C). In HCC, TP53 (17.6\%) and CTNNB1 (11.7\%) were the top two mutations detected with the current platform (Supplementary Fig 1D). In general, the frequencies of somatic mutations detected in this study were comparable to those reported in TCGA. However, the incidence of EGFR mutations (48.6\%) was significantly higher in our study cohort when compared with the reported incidence of EGFR mutations in lung cancer $(\sim 10 \%){ }^{16}$

\section{Molecular profile-based matched treatment}

Of the 342 patients with at least one gene aberration, 31 patients were matched to ongoing clinical trial of a molecularly targeted agent (Fig 1). Of 342 patients, 72 patients received matching molecularly targeted agents in the context of clinical practice. Three patients were assigned to matching targeted agents but did not receive the treatment due to deterioration of the disease. 226 patients did not have molecular aberrations that were matched to currently available treatments either in the context of a clinical trial or in clinical practice and were therefore treated with unmatched cytotoxic or other targeted clinical trial drugs. Ten patients did not have matched drugs available and did not receive any treatment after molecular profiling due to medical conditions (i.e., poor performance).

The primary outcome for this trial was the feasibility of molecular tests and response rate. The response rate for the matched treated group was significantly higher in 
Table 2: Frequency of Molecular Aberrations

\begin{tabular}{|l|c|c|}
\hline \multicolumn{2}{|c|}{ No. of patients } \\
\hline \# of patients consented for molecular analysis & 428 & 100 \\
\hline \# of specimens quality control passed & 407 & \\
\hline No. of aberrations detected for AmpliSeq & & \\
\hline 0 & 65 & 15.9 \\
\hline 1 & 141 & 34.6 \\
\hline 2 & 123 & 30.2 \\
\hline 3 & 51 & 12.5 \\
\hline$\geq 4$ & 27 & 6.6 \\
\hline No. of patients with at least one aberrations by AmpliSeq alone & 342 & 84.1 \\
\hline$\#$ of specimens available for both Ampliseq and 21-gene copy number variation array & 281 & \\
\hline No. of aberrations detected for AmpliSeq and 21-gene copy number variation array* & & \\
\hline 0 & 41 & 14.6 \\
\hline 1 & 79 & 28.1 \\
\hline 2 & 81 & 28.8 \\
\hline 3 & 51 & 18.1 \\
\hline$\geq 4$ & 29 & 10.3 \\
\hline
\end{tabular}

${ }^{*} N=407$ Ampliseq 2.0 +21-gene copy number variation array; $N=281$, Ampliseq 2.0 available

the genome-matched treated group for gastrointestinal/ hepatobiliary/rare tumors (matched vs non-matched, $42.6 \%$ vs $24.3 \%, P=.009)$ and lung cancer cohort (matched vs non-matched, $61.2 \%$ vs $28.6 \%<P=.001$ ) (Figs. 3A and 3B).

\section{Validation of the nanostring CNV 21-gene assay by fluorescence in situ hybridization (FISH), immunohistochemistry (IHC), or quantitative real time PCR (qRT-PCR)}

Next, we validated the results obtained from nanostring 21-gene assays using conventional FISH, IHC, and/or qRT-PCR. In nine selected cases with human epidermal growth factor receptor 2 (HER2) positivity in the nanostring assay, seven cases were concordant with conventional HER2 FISH results (Fig 4A). Two discordant cases exhibited low CNV values in FFPE tissue specimens. For FGFR2 amplification, the concordance rate was $100 \%$ in seven selected cases (Fig 4B). Likewise, the concordance rate for $M E T$ amplification was $100 \%$ in nine selected cases, regardless of the type of tissue specimen (FFPE versus fresh; Fig 4C). Validation data for CCND1, CCNE1, $K R A S, P I K 3 C A, C D K 4$, and MDM2 amplifications are provided in the Supplementary Material. In general, the concordance rate between the nanostring 21-gene assay and conventional FISH or IHC was more than $95 \%$, except for PIK3CA amplification.

\section{DISCUSSION}

In this study, we validated the clinical feasibility of a prospective molecular screening tool with a combination of cancer panel screening and a nanostring 21-gene CNV assay. The success rate of obtaining the molecular profile was extremely high $(95.1 \%, 407 / 428)$ for the cancer panel alone. Given the importance of focal gene amplification, especially in gastrointestinal cancer [5], we designed this screening tool to comprehensively identify actionable mutations and gene amplifications. We were successful in profiling $281(65.7 \%)$ of the enrolled patients to acquire both cancer panel and 21-gene CNV assay data. Importantly, about one-quarter of patients, who underwent molecular screening tests of their tumor samples were matched to a currently ongoing trial or a commercially available drug. The response rate was considerably higher in the genome-matched treated patient cohort when compared with the non-matched treated patient cohort.

Similar trials involving master genomic profiling protocols, which are then matched to available treatments for refractory cancer patients are ongoing. Recently, the interim results of the Molecular Screening for Cancer Treatment Optimization (MOSCATO01) trial were reported in 2013 [17]. From December 2011 to August 2012, 129 heavily pretreated patients (median of three previous treatment lines) consented to participation, and 111 (86\%) had dedicated tumor biopsy samples. An actionable target 
(A)

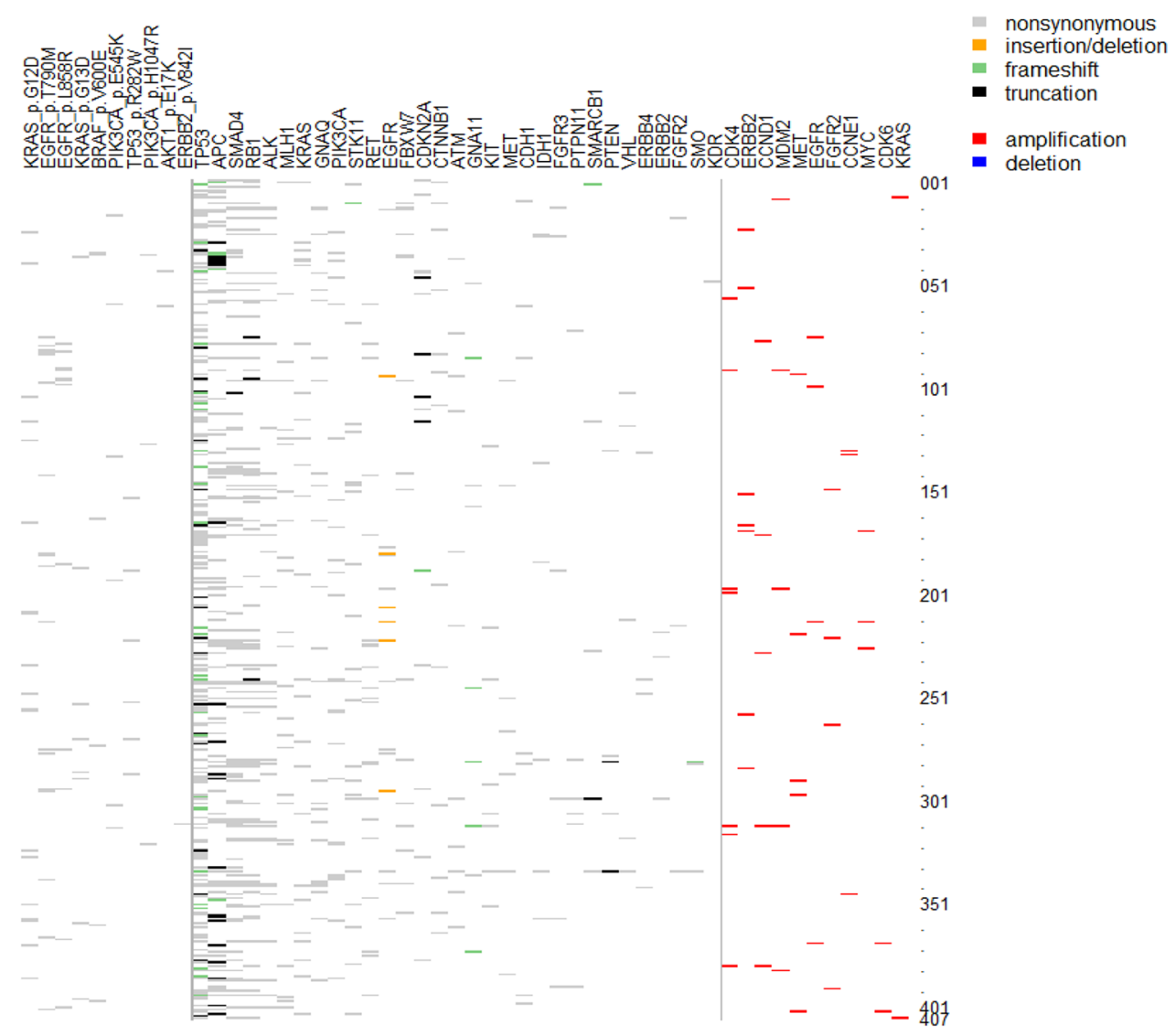

\section{(B) $\mathbf{N}=\mathbf{4 0 7}$}

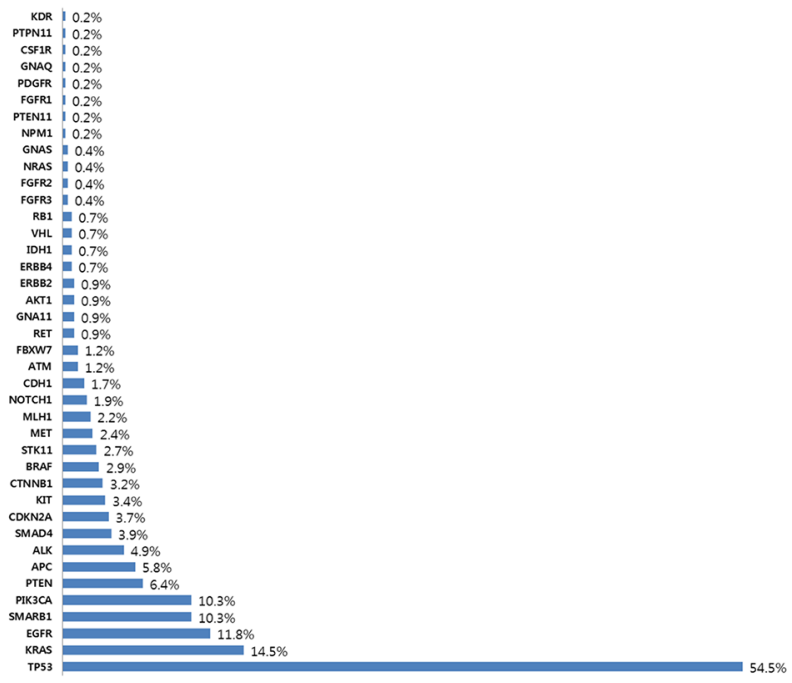

(C) $\mathrm{N}=\mathbf{2 8 1}$

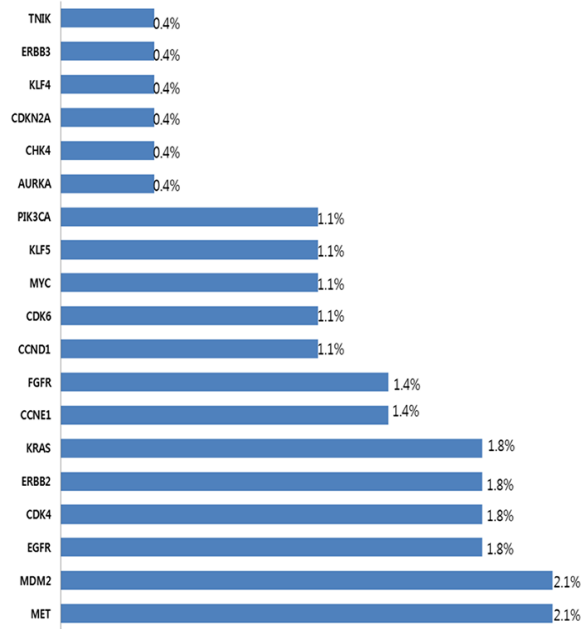

Figure 2: Molecular aberrations in advanced solid tumors. A. Distribution of somatic mutations and copy number variations in 407 solid cancers B. Frequency of somatic mutations $(N=407)$ C. Frequency of copy number variations $(N=281)$

was identified in 52 patients $(40 \%)$, of whom 25 were treated with a matched targeted therapy. In their report, the PFS ratio was greater than 1.3 among 9 out of 19 evaluable patients $(47 \%)$, which led to the conclusion that high- throughput molecular analysis was feasible in daily practice. Notably, the percentage of patients undergoing treatment with a matched molecularly targeted agent based on genomic profiling was similar in our study (24.1\%) and 
Response Rate (\%)

(A)

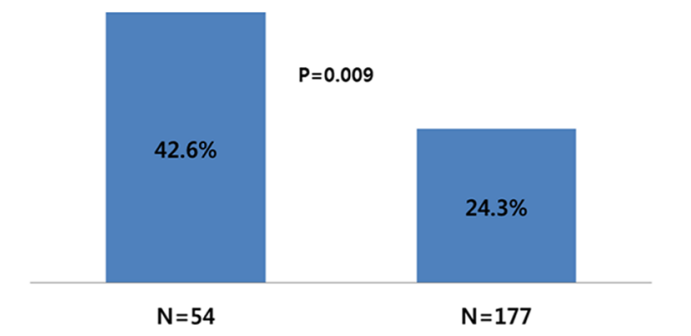

With matched to targeted agents Without matched to targeted agents

\begin{tabular}{cc}
\hline NEXT-1 Trial Cohort, N=231 & N \\
\hline Disease type & 91 \\
\hline AGC & 51 \\
CRC & 15 \\
Sarcoma & 12 \\
HCC & 12 \\
CCC & 7 \\
Melanoma & 7 \\
Pancreas & 7 \\
NET & 4 \\
MUO & 3 \\
RCC & 3 \\
GIST & 3 \\
AoV & 3 \\
Ovary & 2 \\
Bladder & 2 \\
Esophagus & 2 \\
Gall Bladder & 2 \\
Non-melanoma skin & 2 \\
Adrenocortical & 1 \\
Cervix & 1 \\
Duodenal & 1 \\
Others & 3 \\
\hline
\end{tabular}

Response Rate (\%)

(B)

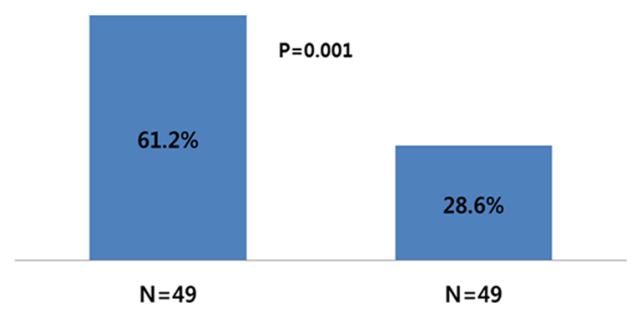

With matched to targeted agents Without matched to targeted agents

\begin{tabular}{lcc}
\hline \multicolumn{2}{c}{ Lung cancer Cohort, N=98 } \\
Disease type & N \\
\hline \multicolumn{2}{c}{ NSCLC (Adenocarcinoma) } & 77 \\
NSCLC (Squamous) & 13 \\
SCLC & 5 \\
Thymic carcinoma & 3 \\
\hline \multicolumn{3}{c}{ Patients detected molecular aberrations } \\
Response & With Matched to & Without Matched \\
& targeted agents & to targeted agents \\
& $(\mathrm{N}=103)$ & (N=226) \\
\hline Complete Response & $0(0.0 \%)$ & $0(0.0 \%)$ \\
Partial Response & $53(51.5 \%)$ & $57(25.2 \%)$ \\
Stable disease & $31(30.1 \%)$ & $91(40.3 \%)$ \\
Progressive disease & $14(13.6 \%)$ & $51(22.6 \%)$ \\
& & \\
Not available & $5(4.9 \%)$ & $27(18.5)$ \\
\hline
\end{tabular}

Figure 3: Efficacy data based on molecular profiling. Response rate according to the matched treatment in the NEXT-1 trial cohorts. A. gastrointestinal/hepatobiliary/rare tumor $(N=231)$ and $\mathbf{B}$. lung cancer cohort $(N=98)$.

the MOSCATO01 trial (25\%). A list of available targeted drugs is provided in Supplementary Table 3.

The SHIVA trial also reported the feasibility of genomic analysis [18]. In this trial, researchers used an NGS platform similar to the one used in our study (AmpliSeq for cancer panel), but used Cytoscan for CNV analysis and an IHC panel including estrogen receptor (ER), progesterone receptor (PR), and androgen receptor (AR). In the SHIVA trial, the success rate for acquiring mutations was $66 \%$, with a tumor content cut-off of greater than $50 \%$. The success rate for the interim analysis of the SAFIR01 study in breast cancer was $71 \%$, with a $50 \%$ threshold for tumor cellularity [19]. Compared with these three large-scale upfront molecular screening trials, the success rate for the NEXT-1/PERSEQ trials was $95 \%$ for the AmpliSeq panel, with a $60 \%$ cut off for tumor content. While the three above-mentioned trials used mandatory fresh tumor biopsies, only $40 \%$ of the specimens were fresh tumors. The success rate for the combined analysis with AmpliSeq and the nanostring CNV panel was 65.7\% in this study. In line with other similar molecular screening studies, the accrual rate for the trial was extremely high (428 patients over 10 months).

Despite the high success rate in genomic profiling and the reasonable rate of patients being treated with matched targeted agents, we need to increase the efficiency of the match rate between genomic data and actual treatment. The number of patients who actually received matched therapy in the context of clinical trials according to the genomic profiling was 31 patients, which should be expanded. Currently, we have opened the VIKTORY (targeted agent eValuation in gastric cancer basKeTKORea studY) trial, a GC-specific screening protocol matched to multiple arms of targeted agents as second-line treatment (NCT\#02299648) based on molecular profiling. In addition, we are increasing the proportion of fresh tumor biopsies in order to expand the genomic sequencing data and to establish patient-derived cells that are linked to the NEXT-1/VIKTORY and PERSEQ trials. We are now adopting hiseq-based targeted sequencing as an expanded 
(A)

\begin{tabular}{|c|c|c|c|c|c|}
\hline $\begin{array}{c}\text { Type of Spe } \\
\text { cimen }\end{array}$ & Nanostring CNV & IHC & FISH & FISH results & Concordance \\
\hline FF & 26 & Positive $(3+)$ & 6.89 & positive & yes \\
\hline FFPE & 68 & Positive $(3+)$ & 13.2 & positive & yes \\
\hline FFPE & 12 & Positive $(2+)$ & 3.84 & positive & yes \\
\hline FFPE & 7 & 0 & 0.98 & negative & no \\
\hline FFPE & 19 & Positive $(3+)$ & 5.54 & positive & yes \\
\hline FFPE & 8 & 0 & 0.85 & negative & no \\
\hline FF & 4 & Positive $(3+)$ & 4.44 & positive & yes \\
\hline FF & 7 & Positive (3+) & 5.64 & positive & yes \\
\hline FFPE & 31 & Positive $(3+)$ & 6.09 & positive & yes \\
\hline
\end{tabular}

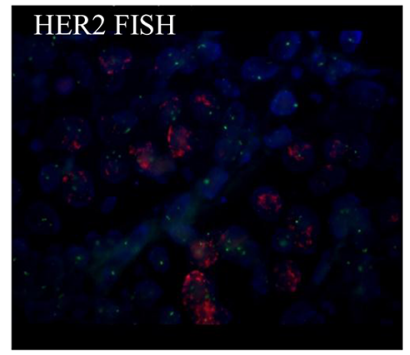

(B)

\begin{tabular}{|c|c|c|c|c|c|c|c|}
\hline Type of specimen & $\begin{array}{c}\text { Clinical } \\
\text { report }\end{array}$ & nanostring CNV & FGFR2 & IHC & FISH & FISH results & $\begin{array}{c}\text { Concord } \\
\text { ance }\end{array}$ \\
\hline FFPE & POS & 35 & 16 & positive & 10.69 & positive & yes \\
\hline FF & POS & 5 & 65 & positive & 10.73 & positive & yes \\
\hline FFPE & POS & 69 & 5 & positive & 3.85 & positive & yes \\
\hline FFPE & POS & 20 & 5 & positive & N/A & positive & yes \\
\hline FFPE & NEG & 2 & 2 & negative & N/A & negative & yes \\
\hline FF & NEG & 2 & 2 & negative & N/A & negative & yes \\
\hline FF & NEG & 2 & 3 & negative & N/A & negative & yes \\
\hline
\end{tabular}

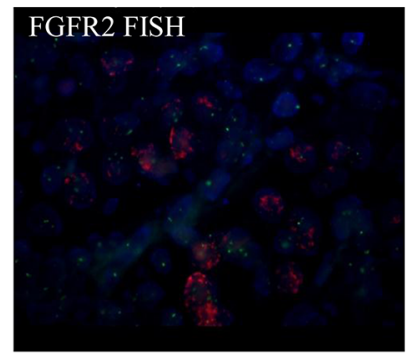

(C)

\begin{tabular}{|c|c|c|c|c|c|c|c|}
\hline $\begin{array}{c}\text { Type of speci } \\
\text { men }\end{array}$ & $\begin{array}{c}\text { clinical } \\
\text { report }\end{array}$ & nanostring CNV & MET & IHC & FISH & FISH results & Concordance \\
\hline FF & POS & 11 & ND & positive & 6.29 & positive & yes \\
\hline FFPE & POS & 15 & ND & positive & 2.5 & positive & yes \\
\hline FF & POS & 11 & ND & positive & 3.58 & positive & yes \\
\hline FFPE & POS & 7 & 4 & positive & 3.91 & positive & yes \\
\hline FF & POS & 7 & 8 & negative & N/A & N/A & N/A \\
\hline FF & POS & 6 & ND & negative & N/A & N/A & N/A \\
\hline FFPE & NEG & 2 & 3 & negative & N/A & N/A & N/A \\
\hline FF & NEG & 2 & 3 & negative & N/A & N/A & N/A \\
\hline FF & NEG & 2 & 3 & negative & N/A & N/A & N/A \\
\hline
\end{tabular}

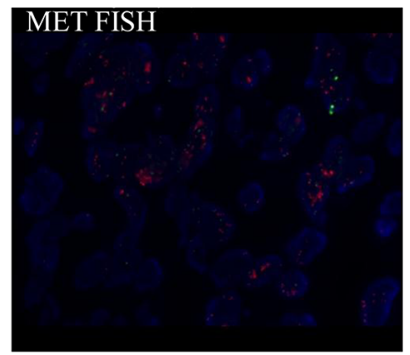

Figure 4: Validation of nanostring CNV 21-gene assay. A. HER2 amplification B. FGFR2 amplification C. MET amplification (left panel, validation summary, right panel, representative FISH results). Abbreviations: CNV, copy number variations; IHC, immunohistochemistry; FF, fresh frozen, FFPE, formalin fixed paraffin embedded tissue; concordance between FISH and nanostring CNV.

cancer panel, which comprises more than 380 genes as a genomic platform. Based on the feasibility results, the NEXT-1 trial will be modified to interrogate the improved treatment outcome based on genome-matched treatment for refractory cancer patients.

Amplification of oncogenes is a major mechanism for gene overexpression and contributes to tumor development in many cancer types [20]. Traditional methods, such as FISH and array comparative genomic hybridization, are limited by the low resolution of genomic regions and high cost and are labor- and time-consuming. nCounter CNV analysis technology is applicable in FFPE specimens, and the results were extensively validated by IHC and FISH for selected genes, including $M E T, M D M 2, C D K 4, H E R 2$, and FGFR2. The results of nCounter CNV analysis were completely concordant with those of IHC and/or FISH for MET, MDM2, FGFR2, and CDK4 (supplementary material). However, discordance was observed for the HER2 probe. One plausible explanation for this discrepancy may be the poor quality of DNA caused by prolonged cold ischemia time. In all, we identified 46 out of 281 cases (16.4\%) with one or more CNV detected through the CNV 21-gene assay. The use of CNV 21-gene assay should be validated further in order to demonstrate a significant increment in identifying matched therapy for metastatic patients. In addition, most of the amplifications that were matched to the actual therapy were gastric cancer. Moreover, we could retrieve CNV results in only 281 patients because high quantity of input DNAs (200ng) required for this assay. Hence, the cost-to benefit ratio to screen all cancer types using nanostring $\mathrm{CNV}$ should be carefully determined. Nevertheless, nanostring CNV 21-gene assay facilitated in identifying patients with gene amplification.

We demonstrate that molecular profiling could benefit patients through identifying matched therapy. The inclusion of metastatic cancer patients with only FFPE tissues available will definitely expand on the spectrum of patients who may potentially benefit from molecular screening. We are now on the second phase of the trial with primary endpoint as Response rate in matched therapy group based on molecular profiling with expanded list of genes when compared with the non-matched therapy and with expanded list of available matched treatment. 


\section{MATERIALS AND METHODS}

\section{Patients}

Patients with metastasis of solid cancers were enrolled onto the NEXT-1 trial [clinicaltrials.gov, NCT\#02141152] and LUNG PERSEQ trial [clinicaltrials.gov, NCT\#02299622] depending on the cancer types at Samsung Medical Center. The study was approved by the institutional review board of the Samsung Medical Center. All study participants provided written informed consent before study entry. Briefly, patients with metastatic solid cancer who may be potentially enrolled onto the matched therapy were eligible to enter the study. Patients with pathologically confirmed cancer and who had either archived tissue or fresh tissues were eligible for genomic analysis. Patients also consented for in vitro establishment of patient-derived cells for research use if tissue was available (this will be reported elsewhere). At the time of genomic analysis, patients were informed of 1) available genome-matched trials, 2) genomematched treatments in practice, and 3) clinical trials or cytotoxic chemotherapies regardless of available genomic data. The list of available matched therapy at the time of patient accrual is listed in Supplementary Table 1.

\section{Tumor samples}

Tumor areas $(>60 \%)$ were dissected under microscopy from 4- $\mu \mathrm{m}$-thick unstained sections by comparison with an H\&E stained slide, and genomic DNA was extracted using a Qiagen DNA FFPE Tissue Kit (Qiagen, Hilden, Germany) according to the manufacturer's instructions. After extraction, we measured concentrations and 260/280 and 260/230 $\mathrm{nm}$ ratios using a spectrophotometer (ND1000, Nanodrop Technologies, ThermoFisher Scientific, MA, USA). Each sample was then quantified with a Qubit fluorometer (Life Technologies, Carlsbad, CA, USA). Samples of genomic DNA with more than 10 ng measured by the Qubit fluorometer were subjected to AmpliSeq library preparation. To identify actionable CNVs, we used a 21-gene nCounter CNV assay, as previously described $[11,15]$. An AmpliSeq cancer panel v2 was examined.

\section{Ion ampliseq cancer panel v2}

We used the Ion AmpliSeq Cancer Panel v2 (Ion Torrent) to detect frequent somatic mutations. Detailed methods were published previously [11]. Briefly, this assay examines 2,855 mutations in 50 commonly mutated oncogenes and tumor-suppressor genes. We used IonTorrent variant caller (version 4.0) for automated data analysis.

\section{nCounter 21-gene CNV assay}

We custom designed a 21-gene CNV assay as outlined in a previous study [11]. We selected 21 genes based on available targeted agents at the time of study design. The 21 genes included AURAKA, CCND1, CCNE1, CDK4, CDK6, CDNK1A, CDNK2A, EGFR, ERBB2, ERBB3, FGFR1, FGFR2, IGFR1R, KLF5, KRAS, MDM2, MET, MITF, MYC, PIK3CA, and TNIK. For detection of CNVs, nCounter Copy Number Variation CodeSets were used with 200 ng purified genomic DNA. DNA was fragmented via AluI digestion and denatured at $95^{\circ} \mathrm{C}$. Fragmented DNA was hybridized with the codeset of 21 genes in the nCounter Cancer CN Assay Kit (Nanostring Technologies) for $18 \mathrm{~h}$ at $65^{\circ} \mathrm{C}$ and processed according to the manufacturer's instructions (Supplementary Table 2) [16]. The nCounter Digital Analyzer counted and tabulated the signals of reporter probes. Quantified data were analyzed using NanoString's nSolver Analysis Software. For normalization of the results, human universal genomic DNA (PR-G3041, Promega) was used. To determine cut off value, we validated nCounter results using conventional FISH, IHC, and/or qRT-PCR and the cut off value was $\geq 3$ in cases using DNAs extracted from fresh tissue and $\geq \mathrm{v} 5$ in cases using DNAs from formalin-fixed paraffin embedded tissue samples. In cases with poor DNA quality, the CNV panel resulted in high value in almost all genes and caution in interpretation was needed. Tumor tissues from 281 patients were available for CNV analysis. In cases with gene amplification identified through the nanostring CNV assay, confirmation using FISH and real-time qRTPCR was conducted (Supplementary Table 3). Detailed protocols for qRT-PCR, IHC, and FISH are provided in the previous study [10].

\section{Analytical methods}

We excluded all synonymous changes after an automated mutation-calling algorithm was used to detect supposed mutations. We used cutoff values of more than $6 \%$ variant frequency and more than X100 coverage to detect true mutational changes in accordance with previous studies and our own experience. We filtered out single-nucleotide polymorphisms after manual review of each polymorphism in the Catalogue of Somatic Mutations in Cancer (COSMIC, http://cancer.sanger.ac.uk/ cancergenome/projects/cosmic). In order to reduce the potential false-positive calls, the following criteria were used to generate final variant calling. The filtering of the variants was performed by analyzing the coverage $(>100$ $\times)$, quality score $(>30)$, and variant frequency of each sample $(>1 \%)$. We also discarded Korean-specific germline variants, such as rs1042522 in TP53 and rs1870377 in KDR. Only functional mutations were included in further analysis. We used the MyCancerGenome database (http://www.mycancergenome.org/) to address clinically actionable mutations. A heat map was generated using $\mathrm{R}$ version 3.0.2 with the gplots package. For wellknown genes mutated in each cancer type, a manual 
review of automated calling results was performed to catch deleterious mutations using the Integrative Genomic Viewer (Broad Institute) with slightly low variant frequency. Patients' characteristics were analyzed using descriptive statistics. Categorical data were described using contingency tables, including counts and percentages.

\section{FUNDING/SUPPORT}

This work was supported by a grant from the Korean Health Technology R\&D Project, Ministry of Health \& Welfare, Republic of Korea (HI13C1951, HI14C2188) and through the Korea Health Industry Development Institute (KHIDI), funded by the Ministry of Health \& Welfare, Republic of Korea (HI14C0072, HI14C3418). Support was also provided by a grant from the 20 by 20 project of Samsung Medical Center (GF01140111).

\section{Author contributions}

Seung Tae Kim, Jeeyun Lee, Mineui Hong and Kyunghee Park served as co-first authors, each with equal contribution to the manuscript

Study concept and design: Jeeyun Lee, Keunchil Park, Kyoung-Mee Kim

Acquisition, analysis or interpretation of data: Seung Tae Kim, Jeeyun Lee, Mineui Hong, Kyunghee Park, Joon Oh Park, Tae Jin Ahn, Se Hoon Park, Young Suk Park, Ho Yeong Lim, Jong-Mu Sun, Jin Seok Ahn, MyungJu Ahn, Hee Cheol Kim, Tae Sung Sohn, Dong Il Choi, Jong Ho Cho, Jin Seok Heo, Wooil Kwon, Sang Won Uhm, Hyuk Lee, Byung-Hoon Min, Sung No Hong, Duk Hwan Kim, Sin Ho Jung, Woongyang Park, Kyoung-Mee Kim, Won Ki Kang, and Keunchil Park

Administrative, technical, or material support: Mineui Hong, Kyunghee Park, Tae Jin Ahn, Kyoung-Mee Kim, Sun Young Kim, Duk Hwan Kim, Sin Ho Jung, Woongyang Park and Jeeyun Lee

Study supervision: Kyoung-Mee Kim, Won Ki Kang, Keunchil Park and Jeeyun Lee

Seung Tae Kim, Jeeyun Lee, Kyoung-Mee Kim, Won Ki Kang and Keunchil Park had full access to all the data in the study and take responsibility for the integrity of the data and the accuracy of the data analysis

\section{ROLE OF THE FUNDER/SPONSOR}

The funders had no role in the design and conduct of the study.

\section{CONFLICTS OF INTEREST}

The authors have declared that no competing interests exist.

\section{REFERENCES}

1. Hammerman PS, Sos ML, Ramos AH, et al. Mutations in the DDR2 kinase gene identify a novel therapeutic target in squamous cell lung cancer. Cancer Discov. 2011; 1:78-89.

2. Wang K, Yuen ST, Xu J, et al: Whole-genome sequencing and comprehensive molecular profiling identify new driver mutations in gastric cancer. Nat Genet 2014; 46:573-582.

3. Wong SS, Kim KM, Ting JC, et al. Genomic landscape and genetic heterogeneity in gastric adenocarcinoma revealed by whole-genome sequencing. Nat Commun. 2014; 5:5477.

4. Meyerson M, Gabriel S, Getz G. Advances in understanding cancer genomes through second-generation sequencing. Nat Rev Genet. 2010; 11:685-696.

5. Cancer Genome Atlas Research Network. Comprehensive molecular characterization of gastric adenocarcinoma. Nature. 2014; 513:202-209.

6. Cancer Genome Atlas Network. Comprehensive molecular portraits of human breast tumours. Nature. 2012; 490:61-70.

7. Kris MG, Johnson BE, Berry LD, et al. Using multiplexed assays of oncogenic drivers in lung cancers to select targeted drugs. JAMA. 2014; 311:1998-2006.

8. Von Hoff DD, Stephenson JJ Jr., Rosen P, et al. Pilot study using molecular profiling of patients' tumors to find potential targets and select treatments for their refractory cancers. J Clin Oncol. 2010; 28:4877-4883.

9. Tsimberidou AM, Iskander NG, Hong DS, et al. Personalized medicine in a phase I clinical trials program: the MD Anderson Cancer Center initiative. Clin Cancer Res. 2012; 18:6373-6383.

10. Rothberg JM, Hinz W, Rearick TM, et al. An integrated semiconductor device enabling non-optical genome sequencing. Nature. 2011; 475:348-352.

11. Kim S, Lee J, Hong ME, et al. High-throughput sequencing and copy number variation detection using formalin fixed embedded tissue in metastatic gastric cancer. PLoS One. 2014; 9:e111693.

12. Singh RR, Patel KP, Routbort MJ, et al. Clinical validation of a next-generation sequencing screen for mutational hotspots in 46 cancer-related genes. J Mol Diagn. 2013; 15:607-622.

13. Beadling $\mathrm{C}$, Neff TL, Heinrich MC, et al. Combining highly multiplexed PCR with semiconductor-based sequencing for rapid cancer genotyping. J Mol Diagn. 2013; 15:171-176.

14. Simon R, Roychowdhury S. Implementing personalized cancer genomics in clinical trials. Nat Rev Drug Discov. $2013 ; 12: 358-369$.

15. Geiss GK, Bumgarner RE, Birditt B, et al. Direct multiplexed measurement of gene expression with colorcoded probe pairs. Nat Biotechnol. 2008; 26:317-325.

16. Calvo E, Baselga J. Ethnic differences in response to epidermal growth factor receptor tyrosine kinase inhibitors. J Clin Oncol. 2006; 24:2158-2163. 
17. Hollebecque A, Massard C, De Baere T, et al. Molecular screening for cancer treatment optimization (MOSCATO 01): A prospective molecular triage trialInterim results. J Clin Oncol. 2013.

18. Le Tourneau C, Paoletti X, Servant N, et al. Randomised proof-of-concept phase II trial comparing targeted therapy based on tumour molecular profiling vs conventional therapy in patients with refractory cancer: results of the feasibility part of the SHIVA trial. Br J Cancer. 2014; 111:17-24.
19. Andre F, Bachelot TD, Campone M, et al. Array CGH and DNA sequencing to personalize targeted treatment of metastatic breast cancer (MBC) patients (pts): A prospective multicentric trial (SAFIR01). J Clin Oncol. 2013.

20. Dai Z, Zhu WG, Morrison CD, et al. A comprehensive search for DNA amplification in lung cancer identifies inhibitors of apoptosis cIAP1 and cIAP2 as candidate oncogenes. Hum Mol Genet. 2003; 12:791-801. 